\title{
Patienten und Dermatologen sollen zueinanderfinden
}

\author{
Die Entfernung von Tätowierungen mittels Laser ist \\ alltäglich geworden - die Suche nach einer kompe- \\ tenten, auf Tattooentfernung spezialisierten Laser- \\ praxis ist dagegen eine Herausforderung.
}

Der Berliner Thomas Sembt hat eine Internetplattform gegründet, die Patienten mit unerwünschten Tätowierungen und Dermatologen zusammenbringen soll. Wer auf „DocTattooentfernung.com" klickt, findet sich schnell zurecht: Ein Menüpunkt enthält Informationen für Ärzte, ein weiterer richtet sich an Patienten. Herzstück der Website ist eine Suchmaschine, welche in ganz Deutschland, Österreich und der Schweiz Laserspezialisten ermittelt. Derzeit sind 25 Experten in der Datenbank hinterlegt. Mit der Plattform wurde ein deutschsprachiges Portal geschaffen, welches es so noch nicht gibt, da es sich um eine Ärztedatenbank handelt, die ausschließlich erfahrene Lasertherapeuten beinhaltet. Sembt geht es darum: „Patienten über die Laserbehandlung aufzuklären und denjenigen, die lieber zum Dermatologen als zum Kosmetiker gehen möchten, eine einfache Suche zu ermöglichen. “ Des Weiteren stehen Austauschmöglichkeiten und Berichterstattung für und von Patienten im Fokus. Es wurde ein Blog entwickelt, wo Erfahrungen, Behandlungsergebnisse und Empfehlungen mit Ärzten diskutiert werden können.

Eine Mitgliedschaft bei „DocTattooentfernung.com“könnte für Dermatologen deswegen interessant sein, weil sie dadurch online auftreten, ihre gewünschte Zielgruppe ansprechen und eine Vernetzung mit anderen Spezialisten stattfindet. Weiter liefert die Plattform Informationen, um mit der Lasertherapie - als Indi-

\section{Nachgefragt: Lasern lernt man nicht am Wochenende}

Herr Sembt, welche Motivation steckt dahinter, dass Sie sich mit dem Thema Tattooentfernung beschäftigen?

Sembt: Meine Motivation nach den echten Tatoo- und Laser-Profis zu suchen, stieg mit dem Respekt vor der Erkenntnis, dass man noch zu wenig über die Tattoo-Mittel und derer Laserentfernung wusste und weiß. Lasern lernt man nicht am Wochenende.

Seit wann gibt es die Plattform?

Sembt: DocTattooentfernung.com gibt es offiziell seit Ende 2012.

Wie hat sich seither das Interesse an der Plattform entwickelt? Verraten Sie uns Ihre Zugriffszahlen?

Sembt: Das Interesse ist immens! Die Zugriffszahlen - und so viel darf ich verraten - nehmen jeden Monat um $50 \%$ zu. Gemessen am Google-Raking sind wir von Seite 28 beim Suchbegriff "Tattoentfernung" auf Seite 3 innerhalb von sechs Monaten katapultiert worden.

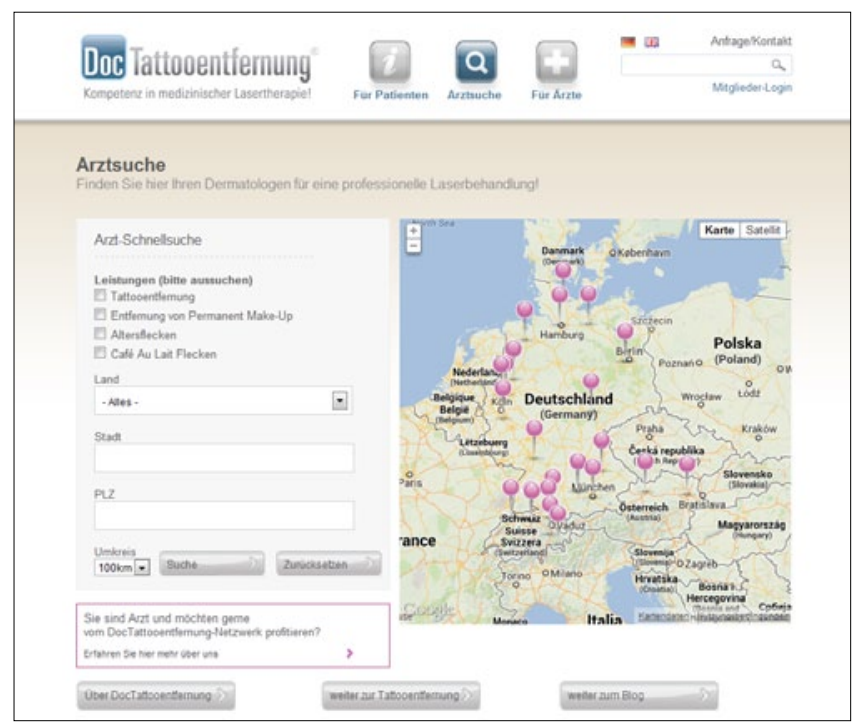

Herzstück von DocTattooentfernung.com ist eine Datenbank, die eine Suche nach Dermatologen ermöglicht, welche auf Tattooentfernung spezialisiert sind.

viduelle Gesundheitsleistung (IGeL) - wirtschaftlich erfolgreich zu sein.

Die Website finanziert sich durch einen monatlichen Mitgliedsbeitrag von 49,-Euro brutto, alternativ können sich die Dermatologen auch in Form von Blog-Beiträgen, zu den Kernthemen Lasertherapie und Pigmententfernung, beteiligen.

\section{sik}

Wie garantieren und prüfen Sie die Qualifikation Ihrer Mitglieder? Sembt: Wir dürfen und können nichts garantieren. Der Laie darf sich auch ohne dermatologische Ausbildung Experte, Profi oder Spezialist nennen. Die Mitglieder der Deutschen Dermatologischen Lasergesellschaft (DDL) hingegen, haben in einigen Fällen ein zusätzliches Studium absolviert, das sogenannte D.A.L.MStudium. Einige derer Absolventen und vor allen Dingen Dozenten werden Sie im Kreise von DocTattooentfernung wieder finden.

Lehnen Sie auch Mitgliedschaften ab?

Sembt: Ganz klares Ja! Und gar nicht so wenige! Wer meine Internetseite als „Verkaufstool" für sein Leistungsspektrum interpretiert, der ist bei mir falsch! Es gibt zwei, drei Zielfragen bei Bewerbungsgesprächen, anhand derer ich diese Leute sofort identifizieren kann. Auch, ob sie Ahnung von ihrer Laserthearpie zur Tattooentfernung haben oder nicht.

Das Interview führte Simone Kohler 